\title{
Crilvastatin 1S
}

National Cancer Institute

\section{Source}

National Cancer Institute. Crilvastatin 1S. NCI Thesaurus. Code C81160.

The $1 \mathrm{~S}$ isomer of crilvastatin, a pyrrolidone non-competitive inhibitor of hydroxymethylglutaryl coenzyme A (HMG-CoA) reductase. 